\title{
All-optical measurement of the complete waveform of octave-spanning ultrashort light pulses
}

\author{
Miguel Miranda, ${ }^{1,2,}$ Francisco Silva, ${ }^{3}$ Lana Neoričl, ${ }^{1}$ Chen Guo, ${ }^{1}$ Vladimir Pervak, ${ }^{4}$ \\ Miguel Canhota, ${ }^{2}$ Ana S. Silva, ${ }^{2}$ Íñigo J. Sola, ${ }^{5}$ Rosa Romero, ${ }^{3}$ Paulo T. Guerreiro, ${ }^{3}$ \\ Anne L'Huillier, ${ }^{1}$ Cord L. Arnold, ${ }^{1}$ and Helder Crespo ${ }^{2,3}$ \\ ${ }^{1}$ Department of Physics, Lund University, P.O. Box 118, SE-221 00 Lund, Sweden \\ ${ }^{2}$ IFIMUP-IN and Departamento de Física e Astronomia, Universidade do Porto, Rua do Campo Alegre 687, 4169-007 Porto, Portugal \\ ${ }^{3}$ Sphere Ultrafast Photonics, S.A., Rua do Campo Alegre 1021, Edifício FC6, 4169-007 Porto, Portugal \\ ${ }^{4}$ UltraFast Innovations GmbH, Am Coulombwall 1, 85748 Garching, Germany \\ ${ }^{5}$ Grupo de Aplicaciones del Láser y Fotónica (ALF), University of Salamanca, Pl. de la Merced s/n, E-37008 Salamanca, Spain \\ ${ }^{*}$ Corresponding author: mmiranda@fc.up.pt
}

\begin{abstract}
We demonstrate the complete temporal characterization of the optical waveform of visible near-infrared octave-spanning ultrashort laser pulses, using an all-optical, all-solidstate and fully inline dispersion-scan device based only on second-harmonic generation. (๑) 2018 Optical Society of America
\end{abstract}

https://doi.org/10.1364/OL.99.099999

Ultrashort light pulses with durations of only a few optical cycles are a unique tool for the investigation and control of matter, enabling access to femto- and attosecond phenomena in atomic, molecular, and condensed matter physics [1]. The carrier-envelope phase (CEP) of few- to single-cycle laser pulses is also relevant in new regimes of relativistic electron acceleration at kilohertz $(\mathrm{kHz})$ repetition rates [2], as well as in other effects, such as plasmonic field enhancement of low-energy few-cycle pulses [3], which have enabled the control of electrons emitted from nanoscale metal tips at the attosecond level. The knowledge and control of the waveform of a light pulse in-situ, i.e., in a given plane where an experiment may take place, either at high or low pulse energies, is highly desirable for achieving reproducible isolated attosecond pulses and for the precise control of matter by the electric field of light at sub-cycle time scales.

The generation of ultrashort pulses has always been directly related to their measurement, which involves knowing the spectral intensity and phase of the pulses. Many self-referenced optical techniques have been developed over the past decades to access the latter (apart from the CEP), such as frequencyresolved optical gating (FROG) and spectral-phase interferometry for direct electric-field reconstruction (SPIDER), which rely on some nonlinear optical effects [4]. To access the CEP, independent measurements based on $f$-to- $2 f$ and related interferometric techniques $[5,6]$ or stereo abovethreshold ionization (ATI) [7] are usually employed. These give information about the stability of the CEP and enable its control and stabilization, but often do not provide its absolute value, preventing knowledge of the exact optical waveform.

There are presently several techniques capable of measuring the full electric field of light pulses based on, e.g., extreme ultraviolet (XUV) -based photoelectron detection [8-10], electrooptic sampling [11], and XUV spectroscopy [12,13]. It is nevertheless highly desirable to extend traditional techniques in the optical domain to include absolute phase measurement capabilities, both for simplicity and sensitivity. Recently, CEPcapable methods based on FROG have been proposed [14,15]. In this paper, we present and demonstrate a further development of the dispersion scan $(d$-scan) technique $[16,17]$, capable of measuring the full electric field of octave-spanning pulses.

The $d$-scan pulse characterization technique relies on measuring the spectrum of a nonlinear signal, such as secondharmonic generation (SHG), as a function of dispersion applied to a single beam. It requires only a pulse compressor (usually already in place), a nonlinear medium, and an optical spectrometer, yet enables precise measurements down to single-cycle durations [18].

In the case of octave-spanning spectra, the fundamental spectrum and the SHG trace overlap, which is usually undesirable. It has however been recognized that this overlap can be used to determine the CEP of a pulse $[14,15]$. This spectral overlap is routinely used to measure CEP variations of ultrashort pulses [5]; however, this usually is not an absolute CEP measurement, since it is done as an independent measurement from the rest of the spectral phase. The basic principle behind our complete characterization of ultrashort pulses is to use an SHG-based characterization technique, where we include the CEP-dependent interference between the fundamental and SHG fields in the phase retrieval. Unlike other approaches that use separate CEP and spectral phase measurements [19], our method allows us to extract the complete phase information and thus reconstruct the optical waveform. 
The concept is depicted in Fig. 1. The pulses are negatively chirped using chirped mirrors (not shown) and go through glass wedges, which introduce a varying glass thickness around the best compression point. After the SHG crystal, a polarizer is placed. In a standard $d$-scan setup, the polarizer would be oriented with the SHG field and block the fundamental field. In this case, however, the polarizer is oriented in such a way that a small amount of fundamental is allowed to pass and interfere with the SHG light. The polarizer also projects the fundamental and SHG fields into a common polarization axis, which is necessary for interference to occur.

The vectorial nature of the electric field has to be taken into account: in our case, where horizontal polarization was used, the direction of the electric field has to be defined (i.e., whether a positive electric field pointing left or right). Since the SHG field is perpendicular to the fundamental field, the quadrant used to project the fundamental and SHG fields together has to be specified, as well as the absolute orientation of the SHG crystal axes. In the present measurements, our results have a sign ambiguity. This, however, can be solved if the orientation of the crystal axes is known.

In a first step of the phase retrieval process, the $d$-scan algorithm is used as usual, neglecting the spectral interference. After that, the retrieved phase is fed into a modified algorithm, and now only the absolute phase is allowed to vary. For this last step, the model has to calculate the generated SHG phase (and not only the intensity, as was the case so far) to simulate the interference fringes, as will be discussed below. The absolute phase (which was the only remaining unknown) is determined by minimizing the error between the measured and simulated fringes in an overlap region.

We describe a pulse in the frequency domain as

$$
\tilde{U}(\omega)=|\tilde{U}(\omega)| \exp i \phi(\omega) .
$$

The classical $d$-scan trace is obtained by adding the glass phase as a function of glass insertion and calculating the corresponding SHG spectra, modeled as

$$
\left|\tilde{U}_{\mathrm{SHG}}(\omega)\right|^{2}=R(\omega)\left|\int \tilde{U}(\Omega) \tilde{U}(\omega-\Omega) \mathrm{d} \Omega\right|^{2},
$$

where $R(\omega)$ is a spectral filter that includes SHG efficiency and phase-matching terms. This approximation works well down to the few-cycle regime $[20,21]$. The main difficulty for this work comes from the need to simulate interference fringes between the fundamental and corresponding SHG, for which we need to be able to simulate the phase of the generated SHG field and not only its spectral intensity.

Following Refs. [20-22], we approximate the nonlinear phase of the generated SHG field by keeping only phase terms. Starting from Eq. (22) in Ref. [21] and discarding amplitude terms, we get

$$
\tilde{U}_{\mathrm{SHG}}(\omega)=i \int \tilde{U}(\Omega) \tilde{U}(\omega-\Omega) \exp i \frac{\Delta k(\Omega, \omega-\Omega) L}{2} \mathrm{~d} \Omega,
$$

where

$$
\Delta k(\Omega, \omega-\Omega)=k_{o}(\Omega)+k_{o}(\omega-\Omega)-k_{e}(\omega) .
$$

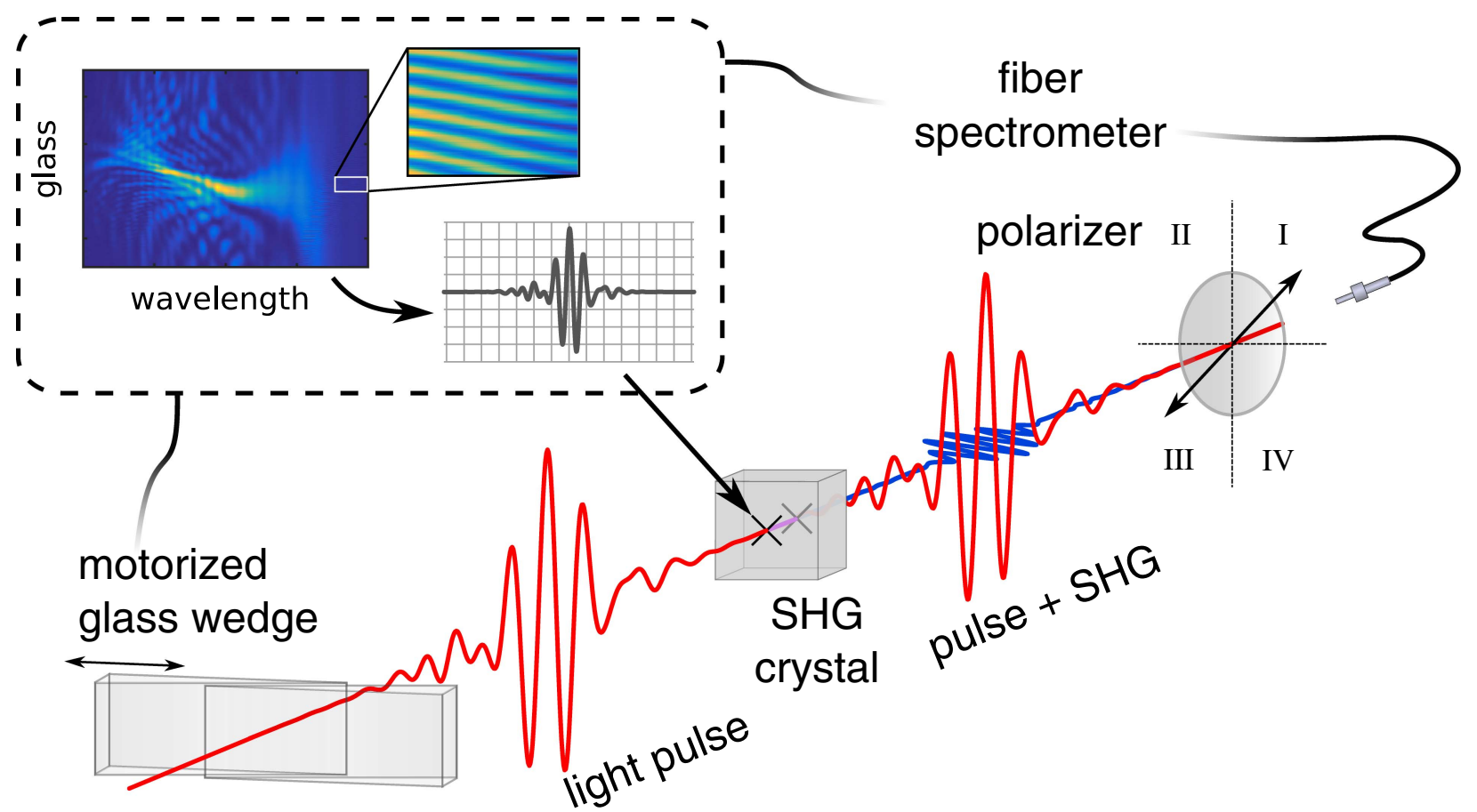

Fig. 1. Concept and simplified schematic of the experimental setup. The pulses are negatively chirped with chirped mirrors (not shown) and go through glass wedges placed on a motorized stage. The pulses are focused on a thin $(4.0 \mu \mathrm{m}) \mathrm{SHG}$ crystal and then go through a wire-grid polarizer, which is used to control the amount of fundamental and SHG and to project both fields into a common polarization axis. The SHG crystal thickness is exaggerated for illustration purposes. The inset shows a typical $d$-scan trace (SHG signal as a function of glass insertion), a zoom of the trace in the spectral interference region, and the corresponding reconstructed optical field. 
By substituting, we get

$$
\begin{aligned}
& \tilde{U}_{\mathrm{SHG}}(\omega) \\
& =i \cdot e^{-i k_{e}(\omega) L / 2} \int \tilde{U}(\Omega) \tilde{U}(\omega-\Omega) e^{i k_{o}(\Omega) L / 2} e^{i k_{o}(\omega-\Omega) L / 2} \mathrm{~d} \Omega,
\end{aligned}
$$

which is a self-convolution of $\tilde{U}(\omega) \exp \left[i k_{o}(\omega) L / 2\right]$ and can be efficiently implemented numerically using a fast Fourier transform. Comparing numerically the phase results obtained this way and using the full integral without approximations, we get errors of only a few milliradians (mrad) for our case of an SHG crystal thickness of $4.0 \mu \mathrm{m}$. The linear phase corresponding to propagation inside the SHG crystal is added to both fields, and the spectral intensity resulting from the interference between them can be calculated.

The pulses were generated using a hollow-core fiber system to spectrally broaden pulses from a multipass Ti:sapphire amplifier (Femtopower, Femtolasers $\mathrm{GmbH}$ ). The amplifier was CEP-stabilized at its output (typical CEP jitter of approximately 280 mrad shot-to-shot [19]). Figure 2 shows measured and retrieved $d$-scan traces, as well as the corresponding retrieved spectral phase and time-domain reconstruction. In the shown measurement, fundamental light was blocked as well as possible with the polarizer. For this first step, the CEP is ignored in the retrieval process. Using our recently developed algorithm [23], retrievals take about $2 \mathrm{~s}$ on a normal desktop computer using a grid of 1024 points in the frequency domain and 300 insertion steps. A mirror on a flip mount, located just before the focusing mirror, steered the beam towards a broadband fiber spectrometer (Avantes AvaSpec, 200-1100 nm), and the fundamental spectrum was recorded. This spectrum was then used by the algorithm in the retrieval. The SHG spectra were measured by the same spectrometer.

To estimate the confidence in the retrieved spectral phase, the retrieval was run multiple times with slightly different starting guesses. In all cases, the root mean square (RMS) error was approximately $1.5 \%$. For each retrieved spectral phase, we simulated the corresponding SHG phase. This gives us an estimate of how accurately at each wavelength we can expect
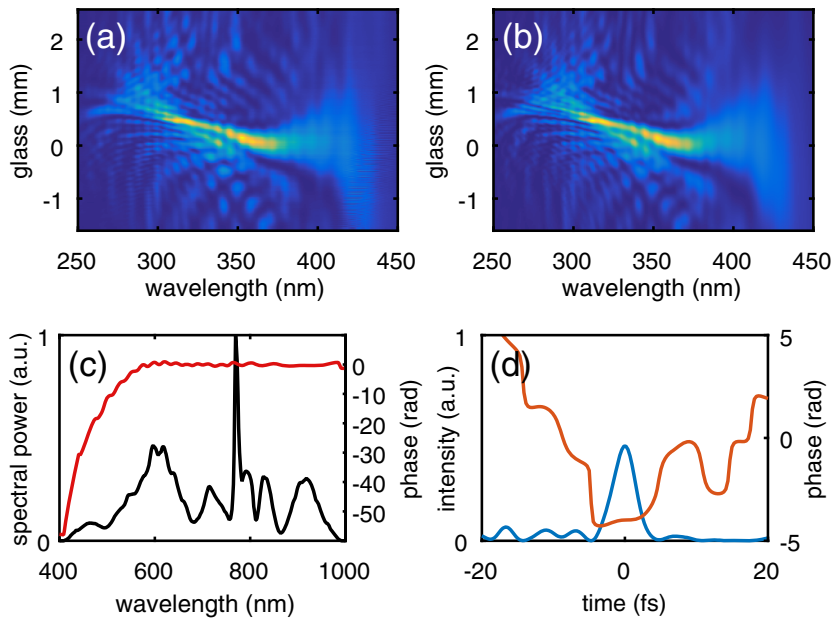

Fig. 2. Typical, a, measured and, b, retrieved $d$-scan traces. c, Measured spectral intensity and retrieved spectral phase with corresponding, d, time-domain reconstruction. The intensity plot in the time domain is normalized to the Fourier limited case.

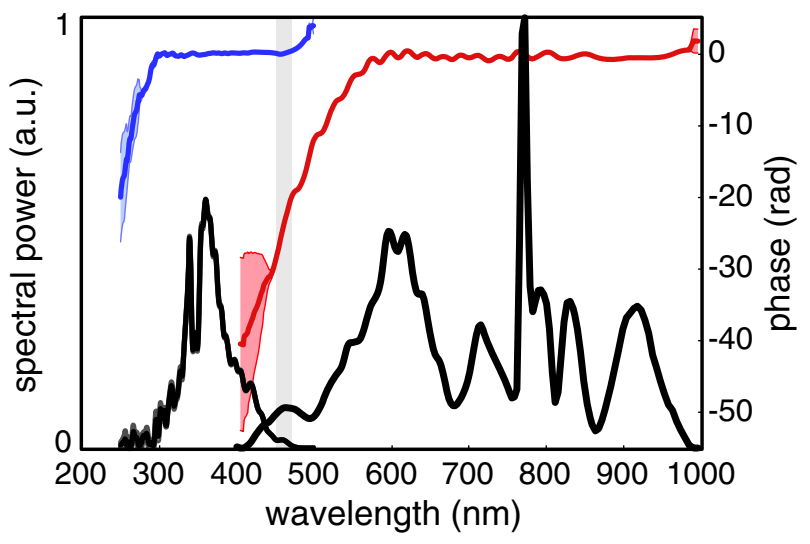

Fig. 3. Measured fundamental spectrum (black line, from 400 to $1000 \mathrm{~nm}$ ) and retrieved fundamental phase (red) and corresponding calculated SHG spectral amplitude (black) and phase (blue), with standard deviations shown in lighter colors. Shaded area (between 450 and $470 \mathrm{~nm}$ ) shows the spectral region, where both fundamental and SHG spectral intensities are non-zero and the corresponding spectral phases are known with high precision.

to simulate the SHG spectral phase. Figure 3 shows the result of this statistical analysis. There is only a narrow spectral region, around $450-470 \mathrm{~nm}$, where (i) the fundamental spectral phase is known precisely, (ii) the SHG phase can be precisely simulated, and (iii) both the fundamental and SHG spectral power
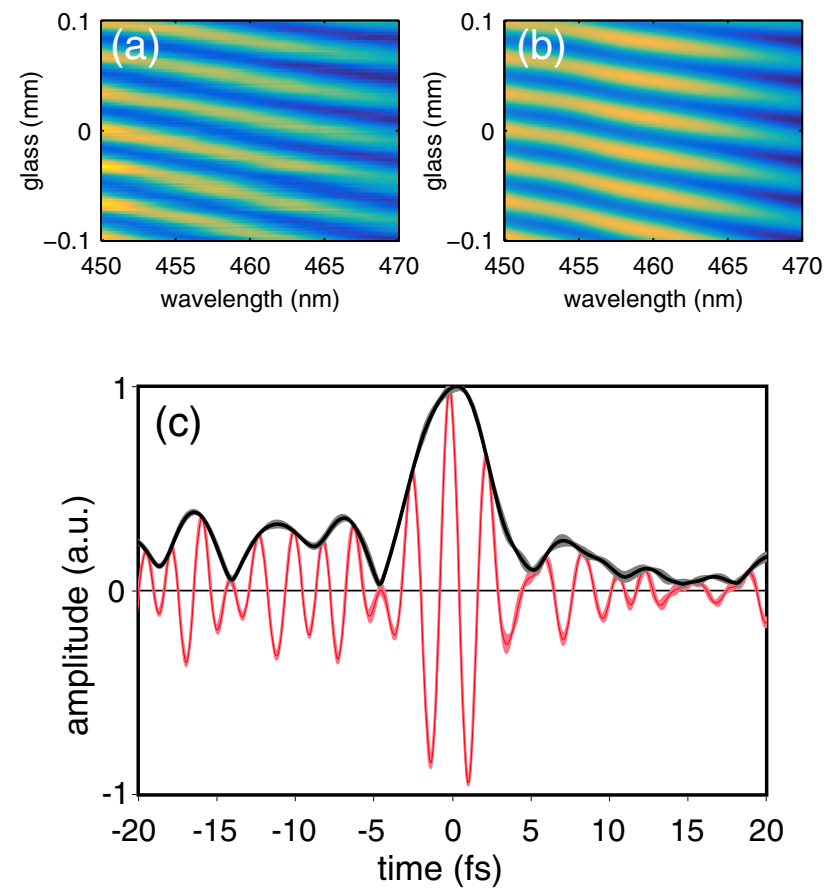

Fig. 4. Close-up of the, a, measured interference fringes in the spectral region between 450 and $470 \mathrm{~nm}$ around the reference glass insertion and, b, corresponding simulated fringes after choosing the absolute spectral phase that minimizes the error in the shown region. After the CEP is determined, that value is input into the previously determined spectral phase, and the full electric field is calculated. c, shows the amplitude (black) and the electric field (red). The lighter shaded areas denote the standard deviation. 
are not zero. The standard deviation on the retrieved phase in the overlap region is about $50 \mathrm{mrad}$.

Figure 4(a) shows the previously discussed interference fringes in the overlap region, measured as a function of glass insertion around the reference insertion. In this case, the polarizer was slightly rotated to transmit a small fraction of the fundamental beam. Taking the previously retrieved pulse, we simulate the corresponding trace and adjust the absolute phase (which was the only remaining unknown) to minimize the error between measured (Fig. 4(a)) and simulated (Fig. 4(b)) fringes. Figure 4(c) shows the reconstruction of the electric field corresponding to the measured spectrum and the retrieved spectral phase. Thin lines correspond to the standard deviation error obtained from the multiple retrievals. Errors associated with pulse instabilities and CEP jitter are not included.

In conclusion, we have presented a $d$-scan-based technique capable of measuring the complete electric field of octave-spanning ultrashort pulses. The technique was demonstrated for the case of a hollow-core fiber compressor, which is representative of state-of-the art systems currently used for high-harmonic generation (HHG). The presented technique could help in closing the present gap between groundbreaking and emerging field-driven petahertz optoelectronics [24] and the existing waveform characterization techniques [7-13] by enabling allsolid-state measurement of the driving optical waveforms. The validity of the complete measurement depends on the validity of the model; therefore, we plan in the future to compare the results with other techniques (e.g., Refs. [10,13]) capable of a more direct measurement.

Funding. H2020 European Research Council (ERC) (PALP, SISCAN); Vetenskapsrådet (VR) ; Knut och Alice Wallenbergs Stiftelse ; Consejería de Educación, Junta de Castilla y León (SA046U16, SA287P18); Ministerio de Economía y Competitividad (MINECO) (FIS2015-71933-REDT, FIS2017-87970-R); Fundaçấo para a Ciência e a Tecnologia (FCT) (Network of Extreme Conditions Laboratories-NECL, NORTE-01-0145-FEDER-022096, PTDC/FISOTI/32213/2017, UID/NAN/ 50024/2013).

\section{REFERENCES}

1. F. Calegari, G. Sansone, S. Stagira, C. Vozzi, and M. Nisoli, J. Phys. B 49, 062001 (2016).
2. D. Guénot, D. Gustas, A. Vernier, B. Beaurepaire, F. Böhle, M. Bocoum, M. Lozano, A. Jullien, R. Lopez-Martens, A. Lifschitz, and J. Faure, Nat. Photonics 11, 293 (2017).

3. M. Krüger, M. Schenk, and P. Hommelhoff, Nature 475, 78 (2011).

4. I. A. Walmsley and C. Dorrer, Adv. Opt. Photon. 1, 308 (2009).

5. M. Kakehata, H. Takada, Y. Kobayash, K. Torizuka, Y. Fujihira, T. Homma, and H. Takahashi, Opt. Lett. 26, 1436 (2001).

6. A. Baltuška, T. Udem, M. Uiberacker, M. Hentschel, E. Goulielmakis, C. Gohle, R. Holzwarth, V. S. Yakovlev, A. Scrinzi, T. W. Hänsch, and F. Krausz, Nature 421, 611 (2003).

7. T. Wittmann, B. Horvath, W. Helml, M. G. Schätzel, X. Gu, A. L. Cavalieri, G. G. Paulus, and R. Kienberger, Nat. Phys. 5, 357 (2009).

8. E. Goulielmakis, M. Uiberacker, R. Kienberger, A. Baltuska, V. Yakovlev, A. Scrinzi, T. Westerwalbesloh, U. Kleineberg, U. Heinzmann, M. Drescher, and F. Krausz, Science 305, 1267 (2004).

9. R. Kienberger, E. Goulielmakis, M. Uiberacker, A. Baltuška, V. Yakovlev, F. Bammer, A. Scrinzi, T. Westerwalbesloh, U. Kleineberg, U. Heinzmann, M. Drescher, and F. Krausz, Nature 427, 817 (2004).

10. F. Quéré, Y. Mairesse, and J. Itatani, J. Mod. Opt. 52, 339 (2005).

11. T. Paasch-Colberg, A. Schiffrin, N. Karpowicz, S. Kruchinin, O. Sağlam, S. Keiber, O. Razskazovskaya, S. Mühlbrandt, A. Alnaser, M. Kübel, V. Apalkov, D. Gerster, J. Reichert, T. Wittmann, J. V. Barth, M. I. Stockman, R. Ernstorfer, V. S. Yakovlev, R. Kienberger, and F. Krausz, Nat. Photonics 8, 214 (2014).

12. K. T. Kim, C. Zhang, A. D. Shiner, B. E. Schmidt, F. Légaré, D. M. Villeneuve, and P. B. Corkum, Nat. Photonics 7, 958 (2013).

13. A. S. Wyatt, T. Witting, A. Schiavi, D. Fabris, P. Matia-Hernando, I. A. Walmsley, J. P. Marangos, and J. W. G. Tisch, Optica 3, 303 (2016).

14. Y. Nomura, H. Shirai, and T. Fuji, Nat. Commun. 4, 2820 (2013).

15. E. W. Snedden, D. A. Walsh, and S. P. Jamison, Opt. Express 23, 8507 (2015)

16. M. Miranda, T. Fordell, C. Arnold, A. L'Huillier, and H. Crespo, Opt. Express 20, 688 (2012).

17. M. Miranda, C. L. Arnold, T. Fordell, F. Silva, B. Alonso, R. Weigand, A. L'Huillier, and H. Crespo, Opt. Express 20, 18732 (2012).

18. F. Silva, B. Alonso, W. Holgado, R. Romero, J. S. Román, E. C. Jarque, H. Koop, V. Pervak, H. Crespo, and I. J. Sola, Opt. Lett. 43, 337 (2018).

19. F. Silva, M. Miranda, B. Alonso, J. Rauschenberger, V. Pervak, and H. Crespo, Opt. Express 22, 10181 (2014).

20. A. Baltuška, M. S. Pshenichnikov, and D. A. Wiersma, Opt. Lett. 23, 1474 (1998).

21. A. Baltuška, M. Pshenichnikov, and D. Wiersma, IEEE J. Quantum Electron. 35, 459 (1999).

22. A. Weiner, IEEE J. Quantum Electron. 19, 1276 (1983).

23. M. Miranda, J. Penedones, C. Guo, A. Harth, M. Louisy, L. Neoričić, A. L'Huillier, and C. L. Arnold, J. Opt. Soc. Am. B 34, 190 (2017).

24. A. Schiffrin, T. Paasch-Colberg, N. Karpowicz, V. Apalkov, D. Gerster, S. Mühlbrandt, M. Korbman, J. Reichert, M. Schultze, S. Holzner, J. V. Barth, R. Kienberger, R. Ernstorfer, V. S. Yakovlev, M. I. Stockman, and F. Krausz, Nature 493, 70 (2012). 